\title{
DE MUJERES Y DIOSAS EN LA RELIGIOSIDAD FENICIA Y PÚNICA ${ }^{1}$
}

\author{
RAQUEL RODRIGUEZ MUÑOZ \\ Madrid
}

Recibido: $15 / 8 / 2012$

Aceptado 08/10/2012

\section{Resumen}

En este trabajo realizaremos un estudio sobre el papel de la mujer en la religión fenicio-púnica y su vinculación con algunas divinidades femeninas asociadas a la fertilidad, lo que ha favorecido la aceptación de premisas que proceden de ámbitos que tradicionalmente se han caracterizado por criticar severamente lo opuesto a lo que se postula. El planteamiento de una nueva visión sobre la controvertida práctica de la prostitución sagrada asociada a estas diosas permite explorar un nuevo ámbito de estudio muy interesante en un afán de cuestionar viejas afirmaciones que no han encontrado respaldo epigráfico y arqueológico evidente.

Palabras clave: papel de la mujer, fertilidad, prostitución sagrada.

\begin{abstract}
In this paper we study the role of women in the Phoenician-Punic religion and its relationship with some female deities associated with fertility, which has favored the acceptance of assumptions that come from areas that have traditionally been characterized by severely criticizing the opposite of what you are applying. The approach of a new view on the controversial practice of sacred prostitution associated with these goddesses to explore a new area of interesting study in an effort to challenge old assertions that have not found support epigraphic and archaeological evident.
\end{abstract}

Keywords: the role of women, fertility, sacred prostitution.

1. Queremos dar las gracias a Angie Simonis por invitarnos a participar en esta edición especial de la revista Feminismos dedicada a La Diosa y el poder de las mujeres. Reflexiones sobre la espiritualidad femenina en el siglo XXI. 

Dentro de la perspectiva de género, la historia de las mujeres se ha desarrollado con fuerza en los estudios sobre la antigüedad, siendo actualmente el volumen de producción enorme. Destacado son los trabajos de pioneras como Mary Gilmore Williams, centrado en las emperatrices sirias, y Grace H. Macurdy, que analizó el papel de las reinas en época helenística, los cuales se sitúan en el punto de partida de los estudios que se desarrollarán a lo largo de toda la segunda mitad del siglo $\mathrm{XX}^{2}$.

A pesar de que en un principio las investigaciones se centraban en documentar el papel político de la mujer en la antigüedad, actualmente la historia de las mujeres está abordando las instancias de influencia pública de las mujeres en esferas distintas a la estatal. Un caso particular lo constituye el ámbito religioso, donde también se aprecia las desigualdades y diferencias jerárquicas de hombre y mujeres. La religión es una esfera de poder, por lo que ambos poderes se encuentran estrechamente unidos. En este sentido, la importancia que adquieren las sacerdotisas es destacada, a pesar de que en muchos casos esa importancia esté subordinada al poder masculino. Las prácticas rituales y el ejercicio del sacerdocio tienen características y funciones diferentes según se trate de hombre o mujeres, aunque ambas se integren en la expresión religiosa colectiva. De este modo, pese a la importancia que adquieren algunas mujeres en la religiosidad antigua, éste papel queda relegado al de los hombres. María Dolores Mirón afirma que ello se evidencia incluso en el ámbito de la divinidad, donde, a pesar de que ciertas diosas ejercen un gran poder y funcionalidad, éstos quedan vinculados al modo de entender lo masculino de lo femenino. Las devociones y rituales de las mujeres contribuyen a reforzar, asumir y reproducir los papeles de género, haciendo al mismo tiempo que ellas se integren, en su diferencia y desigualdad, en la vida cívica de sus comunidades ${ }^{3}$.

2. Williams, Mary Gilmore: «Studies in the lives of Roman Empresses, I: Julia Domna». American Journal of Archaeology 6 (1902), pp. 259-305; MACURDY, Grace H. Hellenistic Queens. A study of women-power in Macedonia, Seleucid Syria and Ptolemaic Egyp, The John Hopkins Press, Baltimore, 1932. En Mirón PÉREZ, María Dolores: «Mujeres y poder en la Antigüedad Clásica: Historia y Teoría Feminista», Saldvie 10 (2010), p. 117.

3. Ibíd, pp. 119-120. 
Bajo este concepto nos parece muy importante realizar un estudio sobre el papel que desempeñó la mujer en la religiosidad fenicia y púnica ${ }^{4}$. Dentro de este estudio cobran especial importancia las fuentes escritas ${ }^{5}$ como modo de acercarnos a la práctica cultual fenicia en la que las mujeres participaban. Esta tarea es ardua y complicada ya que, de modo general, la visión que encontramos en las fuentes es ambivalente, cuando no negativa ${ }^{6}$. Para el caso de la mujer, además, suele ser escasa, ya que los datos que se describían afectaban, mayoritariamente, a los hombres. Lancelloti destaca al respecto que

[...] come quella di tante altre donne dell'antichità, anche la vita della donna fenicia si svolgeva, dall'inizio alla fine, prevalecentemente, se non esclusivamente, nell'ambito della familia ed è eventualmente solo in qualche caso, come ad esempio nella vita religiosa (si pensi in particolare alle preferenza devozionali delle singole persone), che può emergeré per barlumi un universo femminile altrimenti difficilmente riconstruibile ${ }^{7}$.

Tratar de constatar arqueológicamente estas prácticas asociadas al mundo femenino en el marco de un culto determinado también es difícil. Un recorrido por los distintos yacimientos en los que se han evidenciado el culto a divinidades fenicias y púnicas no nos permite determinar su posible vinculación con un comportamiento cultual claramente femenino como parte de la práctica religiosa de la sociedad general, en el que el protagonismo era ejercido por el hombre.

La única documentación que podría dar una idea sobre este papel procede de las representaciones iconográficas y algunas de ellas, como veremos, no están exentas de polémica. La facilidad con la que son interpretadas algunas

4. El tema del papel de la mujer en el ámbito religioso fenicio-púnico ya ha sido tratado de manera monográfica por A. M. Jiménez Flores. En este sentido, véase JiMÉNEZ FLORES, Ana María: «La mano de Eva: las mujeres en el culto fenicio-púnico», en EsCACENA Carrasco, José Luis, Ferrer Albelda, Eduardo (eds.): Entre dioses y los hombres: el sacerdocio en la antigüedad. Sevilla, SPAL Monografías VII, 2006, pp. 83-102. Esta autora también ha estudiado el ejercicio del sacerdocio femenino dentro de la práctica religiosa fenicia en JiMÉNEZ FLORES, Ana María. «El sacerdocio femenino en el mundo feniciopúnico», SPAL, no 11, 2002, pp. 9-20.

5. De modo general, para el estudio de la religiosidad fenicia y púnica es obligatoria la consulta de los textos de Herodoto, Polibio, Diodoro Sículo, Plutarco, Apiano, Estrabón, Luciano, Tito Livio, Silio Itálico, Filón de Biblos, Pausanias y Flavio Josefo, así como la del conjunto de relatos que conforman el Antiguo Testamento.

6. DE GEUS, Cornelius H. J., «The material culture of Phoenicia and Israel», en LIPINSKI, Edward (ed.), Phoenicia and the Bible, Studia Phoenicia XI, Leuven, Orientalia Lovaniensia Analecta, no 44, 1991, p. 16.

7. LanCellotti, Maria Grazia, «La donna», en Zamora, J. A. (ed.), El hombre fenicio. Estudios y materiales, Roma, CSIC, 2003, p. 187. 
imágenes, apoyándose en las siempre controvertidas fuentes escritas, evidencia el gran camino que aún queda por recorrer dentro de la bibliografía fenicia y púnica en cuanto al papel de la mujer en el contexto religioso. Esta bibliografía tradicionalmente siempre había apuntado que a las mujeres, dentro de la religión fenicia y púnica, le estaban reservadas principalmente tres prácticas $^{8}$ : la prostitución sagrada, el matrimonio sagrado o la hierogamia y la ofrenda de la virginidad en el templo de las mujeres casaderas, todas ellas vinculadas directamente con la fertilidad ${ }^{9}$. Sobre la primera de ellas, la prostitución sagrada, en los últimos años los investigadores ${ }^{10}$ suelen negar la realización de esta práctica, entendiéndolo como un recurso literario usado por los escritores greco-latinos para desacreditar las costumbres de estos pueblos próximo-orientales. En cuanto a la hierogamia o matrimonio sagrado, ésta se trataba de la unión física entre el rey y la divinidad, la cual era simbolizada por una sacerdotisa. No se trataría de una práctica exclusiva fenicia sino más bien de un comportamiento ritual próximo oriental que únicamente conocemos a través de los textos, textos que también nos informan sobre la ofrenda de la virginidad.

A lo largo de este trabajo trataremos de llamar la atención sobre el papel que ejercía la mujer dentro de la religiosidad fenicia y púnica, exponiendo las continuas afirmaciones que se han escrito al respecto y planteando, en algunos casos, una visión distinta que cuestiona el desarrollo de determinadas prácticas desde el punto de vista estrictamente religioso. Del mismo modo, trataremos de vincular dicho papel femenino con algunos cultos a divinidades cuyos atributos permitían atribuir mayor protagonismo a la mujer.

8. LIPINSKI, Edward, Dieux et Déesses de l’ Únivers phénicienne et puníque, Leide, 1995, pp. 486-487.

9. YAmauchi, Edwin Maseo: «Cultic Prostitution», Orient and Occident. Essays presented to Cyrus H. Gordon on the Occasion of his Sixty-fith Birthday, AOAT 22, Kevelaer-Neukirchen-Vluyn, 1973, p. 213; HensHaw, Richard A., Female and Male. The cultic Personnel: the Bible and the Rest of the Near East, Allison Park, P A, Pickwick Publications, 1994, pp. 191-270, sobre todo 218 y ss.; JiMÉNEZ FLORES, Ana María. El sacerdocio femenino... op. cit., pp. 17-18.

10. Budin, Stephanie Lynn, «Sacred Prostitution in the First Person», en FaraOne, Christopher, MCCluRe, Laura (eds.), Prostitutes and Courtesans in the Ancient World. Madison, 2006, pp. 77-92; Budin, Stephanie Lynn, The Myth of Sacred Prostitution in Antiquity, New York, Cambridge University Press. 2008. Este libro comienza con la afirmación por parte de la autora de que la prostitución sagrada nunca existió en el Próximo Oriente antiguo (p. 1). A lo largo de diez capítulos Stephanie desmonta todas las teorías y datos en los que se ha basado la existencia de esta práctica, a la que considera como un mito.

Feminismo/s 20, diciembre 2012, pp. 143-164 
Como ya hemos señalado, contamos con pocas referencias sobre el papel de la mujer en la sociedad fenicia y púnica, realidad que se hace más patente para el ámbito religioso, donde las fuentes atribuyen el papel protagonista al hombre, al que se le tiene encomendado el sacerdocio y la organización administrativa, económica y cultual del templo ${ }^{11}$, aspecto éste que era común en toda la franja sirio-palestina desde el II milenio ${ }^{12}$. Se trataba de personal especializado, fundamentalmente masculino, que se encontraba al servicio de los dioses ${ }^{13}$. En el ámbito fenicio y púnico formaban parte del mismo los altos estamentos sociales y políticos, algunos de los cuales, en época fenicia oriental, podrían haber compaginado el sacerdocio con el ejercicio de la administración y la política de la monarquía ${ }^{14}$. La inscripción referente al registro de las cuentas del templo de Astarté en Kition (CIS 86A y B) ${ }^{15}$ describe todo un conjunto de personal que estaría al servicio del templo, como acólitos,

11. Sobre el sacerdocio fenicio resulta indispensable el trabajo de Amadasi Guzzo. En este sentido, véase AMADASI GUZZO, Maria Giulia: «Il sacerdote», en ZAMORA, José Ángel (ed.), El hombre fenicio: estudios y materiales, Roma, CSIC, 2003, pp. 45-53. También Jimenez Flores, Ana María, MARín Ceballos, María Cruz: «Jerarquía social y sacerdocio fenicio-púnico: apuntes sobre su relación», en HERNÁNDEZ GUERRA, L. y Jaime Alvar EZQUERRA, (eds.): Jerarquías religiosas y control social en el mundo antiguo. Actas del XXVII Congreso Internacional Girea-Arys IX, Valladolid, 2004, pp. 80-86.

12. En Ugarit, durante el II milenio, la casta sacerdotal la formaban los llamados «hombres del rey», que estarían constituidos por trabajadores y profesionales al servicio del rey que eran sostenidos por el propio palacio. En este contexto, el rey era el sacerdote principal. No contamos con documentos que nos informen sobre la función de la mujer dentro de las prácticas cultuales hasta la época fenicia. Véase ZAMORA, José Ángel: «El sacerdocio en el Levante próximo-oriental (Siria, Fenicia y el mundo púnico): las relaciones entre el culto y el poder y la continuidad en el cambio», en ESCACENA CARRASCO, José Luis y Eduardo FerRer Albelda, (eds.): Entre dioses y los hombres: el sacerdocio en la antigüedad. SPAL Monografías VII, Sevilla, 2006, pp. 63-65.

13. LIPINSKI, Edward, op. cit., p. 451.

14. ElAYI, Josette, «Le roi et la religion dans les cités phéniciennes à l'époque perse», en Religio Phoenicia, Studia Phoenicia IV, Namur, 1986, pp. 253 y ss.; Ribichini, Sergio: «Beliefs and Religious Life», en Moscati, Sabatino (ed.): The Phoenicians, LondonNew York, I. B. Tauris, 2001, pp. 120-153; LIPINSKI, Edward, op. cit., p. 451; ZAMORA, José Ángel, op. cit., pp. 70-77. Este autor señala también la importancia del personal del templo que acompañaría a los sacerdotes en el desarrollo del culto, el cual desempeñaría funciones específicas, así como la posibilidad de que existiese una jerarquía sacerdotal, encabezada por un «jefe de sacerdotes», rb hnm, con diferentes grados sacerdotales. También JiMÉNES FLORES, Ana María: El sacerdocio femenino..., op. cit., pp. 11-13; Jimenez Flores, Ana María, Marín Ceballos, María Cruz, op. cit., p. 78.

15. MASSON, Olivier, SZNYCER, Maurice, Rechercher sur les Phéniciens à Chypre, Ginebra-Paris, Centre de Recherches d'Histoire et de Philologie, 1972, pp. 21-68; CAUBET, Annie: «Les sanctuaires de Kition à l'époque de la dynastie Phénicienne», Religio Phoenicia, Studia Phoenicia IV, Namur, 1986, pp. 155-159. 
panaderos, peluqueros, un maestro del agua ${ }^{16}$, etc., personal destinado a auxiliar a los sacerdotes o sumos sacerdotes y facilitar el correcto desarrollo del culto $^{17}$.

Algunas mujeres vinculadas a la monarquía oriental también tenían cargos relevantes dentro de la religión fenicia, ostentando el papel de sacerdotisa khnt en aquellos cultos asociados a deidades femeninas ${ }^{18}$, como nos ponen de manifiesto algunas inscripciones ${ }^{19}$. A pesar de ello, el dato más antiguo que se conoce no porta el título real ${ }^{20}$, y correspondería a una inscripción funeraria documentada en una crátera fenicia del siglo VIII a. C. de procedencia desconocida que menciona a una mujer como sacerdotisa de Astartée $e^{21}$ aunque algunos investigadores han planteado la posibilidad de que pudiera tratarse de una prostituta sagrada ${ }^{22}$. Más clara resulta la inscripción del sarcófago de Eshmunazor II de Sidón, que nombra a su madre como la portadora de este

16. Este cargo ha sido estudiado de manera monográfica por M. Yon en su trabajo «Le «Maître de l’eau» à Kition», publicado en 1982 formando parte del libro Archéologie au Levant. Recueil R. Saidah. Collection de la Maison de l'Orient Méditerranéen 12, Série archéologique 9, Lyon, pp. 251-263. La importancia del agua en la religiosidad antigua ha sido muy destacada, sobre todo asociada a cultos vinculados a la fertilidad, lo que ha permitido ponerla en relación, para el caso fenicio-púnico, con divinidades como la propia Astarté. En este sentido, véase RodRíGUEz MuÑOz, Raquel: «El uso cúltico del agua en el mundo fenicio y púnico. El caso de Astarté en Cádiz». Herakleion 1, 2008, pp. 29 y ss. A pesar de ello, desconocemos si este cargo existiría en todos los santuarios dedicados a esta diosa.

17. En 1994 R. A. Henshaw dividía en cinco los grupos en los que se podía estructurar el personal encargado del templo y del culto en el ámbito oriental. De este modo, este autor apuntaba un primer grupo, compuesto por altos funcionarios, encargados de liderar el culto, grupo al que le seguiría otro formado por cantantes, músicos y bailarines, un tercero constituido por personal encargado de la magia y las adivinaciones, un cuarto donde tendrían cabida personal destinado a actividades sexuales vinculadas al ámbito de la fertilidad, y un quinto que englobaría al resto de personal auxiliar del templo. En este sentido, véase HENSHAW, op. cit., p. 10.

18. Sobre esta cuestión debemos destacar el artículo ya señalado de A.M. Jiménez Flores dedicado exclusivamente al papel de la mujer como sacerdotisa. En este sentido, véase nota 2.

19. Este papel protagonista de algunas mujeres en el desarrollo del culto se puede rastrear desde momentos del Bronce Final, donde ya encontramos a personajes como Ummahnu, que podría haber formado parte de la casta sacerdotal dentro del culto a la Señora de Biblos. VIDAL, Jordi, «Ummahnu, sierva de la Señora de Biblos. Apuntes prosopográficos», Aula Orientalis 28, 2010, pp. 85-92.

20. LANCELlOTTI, Maria Grazia: Op. cit., p. 193

21. PUECH, Emile. «Un cratère phénicien inscrit: rites et croyances». Transeuphatène 8 (1994), pp. 47-69; AMADASI, op. cit., p. 46; JIMÉNEZ, El sacerdocio femenino..., op. cit., p. 14; JiMÉNEZ, La mano de Eva..., op. cit., p. 85.

22. PUECH, op. cit., pp. 52-53.

Feminismo/s 20, diciembre 2012, pp. 143-164 
cargo excepcional ${ }^{23}$. Destacable también son la inscripción procedente de la necrópolis de Ard-el-Kheraib ${ }^{24}$, donde se menciona a Hanibaal como sacerdotisa, y el epitafio de Sofonibal hkhnt, fechado en época helenística, que también la identifica como tal ${ }^{25}$.

En el ámbito occidental, el sacerdocio era ejercicio por las clases elevadas y las autoridades políticas locales ${ }^{26}$, que, para Edward Lipinski, podían haber ejercido la función de «resucitador de la divinidad ${ }^{27}$, siendo también hereditaria en algunos $\operatorname{casos}^{28}$. Para el caso de nuestro estudio, existen contados ejemplos que mencionan a mujeres que podrían haber ejercido el sacerdocio, todas ellas relacionadas con las clases elevadas de la sociedad, ya que este tipo de actividad, sumamente importante por cuanto supone el vehículo de transmisión de los anhelos y solicitudes de una comunidad en pro de una divinidad por medio de las prácticas rituales sagradas, estaba reservada a los estamentos más elevados de la sociedad. Estos ejemplos proceden en su casi totalidad de contextos funerarios y se reducen a diez inscripciones en las que se mencionan a sacerdotisas ${ }^{29}$. Del mismo modo, existen evidencias

23. La inscripción CIS I, 3= KAI 14 reza «Aquí es que yo Eshmunazor, rey de Sidón, y mi madre Emashstar, sacerdotisa de Astarté nuestra señora, la reina». El sarcófago, fechado en el primer cuarto del siglo $\mathrm{V}$ a. C., está realizado en basalto negro y su manufactura es claramente de tradición egipcia. En AMADASI, op. cit., p. 45. Se han apuntado algunas propuestas que intentan explicar si la detención de este alto cargo cultual, que se trataba de un cargo temporal, no heredado por ninguna mujer de la generación anterior ni hereditable en otras mujeres de la generación siguiente, ejercido por la «tutora» del monarca durante la minoría de edad de éste, fue llevado a cabo como consecuencia de una acto privado o fruto de esa situación de regencia y tutela señalada. En este sentido, véase Elayi, op. cit., p. 255; BONnET, Corinne, Astarté. Dossier documentaire et perspectives historiques, Roma, Consiglio Nazionale delle Ricerche, 1996, p. 33. También JIMÉNEZ El sacerdocio femenino..., op. cit., p. 13.

24. Se trataría de la inscripción CIS I, 5987. Esta necrópolis fue estudiada y publicada en 1909 por A. Merlin y L. Drappier en un trabajo titulado La necròpole puníque d'Ard ElKheraïb à Carthage (Notes et documents publiès par la Direction des Antiquités de Tunis. III), Paris. En 1982 H. Bénichou-Safar las recogió en su estudio sobre las necrópolis de Cartago: Les Tombes Puniques de Carthage. Topographie, structures, inscriptions et rites funéraires, Paris, CNRS.

25. CIS 5950= KAI 93. En AMADASI, op. cit., p. 50.

26. JIMÉNEZ Y MARÍN, op. cit., p. 79 y ss.

27. LIPINSKI, Edward, op. cit., pp. 454-455.

28. RiBichini, op. cit., p. 136.

29. Se trata de las inscripciones CIS I 5940, 5941, 5942, 5947, 5949, 5950, 5961, 5985 y 5994. En LANCELlOTTI, op. cit., p. 193. También la inscripción EH 72. En JiMÉNEZ, La mano de Eva..., op. cit., pp. 86-87. Las inscripciones cartaginesas en las que una mujer ocupa el cargo de sacerdotisa han sido recogidas recientemente por L. A. Ruiz en un trabajo más amplio en el que se presentan aquellas otras que permiten identificar la diversificación social de la propia Cartago. En este sentido, véase RUIZ CABRERO, Luis Alberto: «Sociedad, jerarquías y clases sociales de Cartago», en COSTA, Benjamin; 
de inscripciones en las que se indica su pertenencia al sumo sacerdocio ( $r b$ $k h n t)$, como jefa de las sacerdotisas ${ }^{30}$. Siguiendo estos datos, Jiménez Flores afirma que deben distinguirse entre las "profesionales del culto», formadas y especializadas en la liturgia, y las sacerdotisas como tal, detentadas por las clases elevadas ${ }^{31}$. Este sacerdocio femenino, tal y como dejan entrever estas inscripciones, debió estar asociado al culto de determinadas divinidades, concretamente deidades femeninas con unos marcados atributos vinculados a la fertilidad, la agricultura y la maternidad.

La documentación iconográfica también ha sido tenida en cuenta por los investigadores para tratar de averiguar para el comportamiento y los atuendos de estas administradoras de culto. En el Museo Nacional de Cartago se conserva el sarcófago de Arishutbaal, esposa de Milqarthalos, identificada como una sacerdotisa. Se ha fechado en los siglos IV-III a. C., y, a priori, nos podría dar una idea de como sería el atuendo de estas profesionales ${ }^{32}$. Determinados autores han llamado también la atención sobre algunas estelas votivas con representaciones de lo que podrían ser sacerdotisas ataviadas con largas túnicas en actitud sagrada. Tal es el caso de tres estelas procedentes del tofet de Salambó ${ }^{33}$, Lilibeo ${ }^{34}$ y Mozia ${ }^{35}$. Parece evidente que las mujeres representadas estarían llevando a cabo algún tipo de actividad sagrada o ritual, pero no

FERNÁNDEZ, Jordi H. (eds.), Instituciones, demos y ejército en Cartago. TMAEF 64, 2009, p. 22.

30. Se trata de las inscripciones CIS I 5988, 5949 y EH 67. En LANCELlOTI, op. cit., p. 193; JIMÉNEZ, La mano de Eva..., op. cit., pp. 86-87; RUIZ CABRERO, op. cit., pp. 54-55.

31. JIMÉNEZ, El sacerdocio femenino..., op.cit., p. 14; JIMÉNEZ, La mano de Eva..., op. cit., pp. 85-87.

32. LIPISNKI, op. cit., p. 457.

33. En esta estela aparece a una figura femenina cubierta con una túnica que presenta la mano derecha alzada y la mano izquierda con una píxide. Esta figura se sitúa junto a lo que parece ser un altar en el que se halla una cabeza de toro, lo que ha determinado que tal figura femenina sería una sacerdotisa que estaría llevando a cabo un ritual de sacrificio en honor de una deidad, posiblemente relacionada con la fertilidad, determinación que en este caso parece respaldada por la inscripción de la propia estela. JIMÉNEZ, El sacerdocio femenino..., op.cit., pp. 16-17; JIMÉNEZ, La mano de Eva..., op. cit., p. 88.

34. La mujer que está representada en esta estela también está ataviada con una larga túnica, portando en su mano izquierda una píxide cuyo contenido es vertido en un thymiaterion con el signo de Tanit. En un segundo caso, la posible sacerdotisa porta en su mano izquierda un recipiente y levanta su mano derecha frente a un caduceo. Esta representación también está presidida por el signo de Tanit. BISI, Ana María. «Le stele puniche di Lilibeo e il problema dell'influenza semitica nella religione e nell'arte della Sicilia Occidentale» Karthago XIV (1968), pp. 227-228, Tav. I- II; JiMÉNEZ, La mano de Eva..., op. cit., p. 88.

35. En este caso, se representan dos figuras femeninas que parecen acercarse a depositar una ofrenda en un thymiaterio en un espacio de culto evidenciado por un caduceo y dos signos de Tanit. Ibid., p. 228, Tav.III, 1; Ibidem, p. 88. 
estamos seguros de si, a través de esas representaciones, podemos establecer que en todos los casos se tratasen de verdaderas sacerdotisas y no simples devotas o adoradores de la divinidad. A. Maes ${ }^{36}$, en su trabajo sobre las estelas de Umm el-Amed, llamó la atención sobre la problemática que supone identificar sacerdotes y sacerdotisas a través de las vestimentas de algunas estelas en las que no existe ninguna inscripción que lo corrobore, siendo complicado, por tanto, saber si se trata de representaciones de sacerdotes/sacerdotisas o de fieles, llegando a afirmar que «le costume représenté sur les stèles, aussi bien celui des hommes que celui des femmes, ne suffit pas pour distinguer les prêtres des fidèles $»^{37}$. Tras realizar un análisis sobre la información existente en la Biblia y la observada en los relieves de las estelas, con los puntos en común entre ambas, concluye que «toutes ces observations suffisent à montrer que l'étude des vêtements chez les peuples anciens es assez complexe et qu'il est dès lors dangereux de tirer des conclusions aussi bien des textes sans image que des images sans texte» ${ }^{38}$.

Con ello simplemente queremos expresar la complejidad que supone identificar un determinado personaje en función de su vestimenta y su actitud sin la ayuda de una inscripción que así lo confirme, complejidad que aumenta para el caso que aquí nos ocupa, ya que los datos con los que contamos son aún más escasos.

Dejando a un lado este tema, exceptuando los ejemplos en lo que la mujer ejerce un papel destacado como sacerdotisa durante el ejercicio de prácticas relacionadas con el culto, en líneas generales, el papel de la mujer en la religión fenicia y púnica estaba vinculado, de modo general, a la realización de tareas y prácticas auxiliares al propio culto, aunque no por ello menos importantes. Quizá la más conocida y controvertida sea la prostitución sagrada, práctica asociada a determinadas divinidades que era ejercida principalmente por algunas mujeres, aunque también por hombres ${ }^{39}$. Según Edwin

36. MAES, Annemie: «Le costume phénicien des stèles D Umm el-Amed», en LiPINSKI, Edward (ed.): Phoenicia and the Bible, Studia Phoenicia XI, Orientalia Lovaniensia Analecta 44, Leuven, 1991, pp. 209-230.

37. Ibid., p. 224.

38. Ibid., p. 230.

39. Sobre el tema de la prostitución sagrada imprescindibles son los trabajos de YAMAUCHI, op. cit., pp. 213-222; DELCOR, Mathias, «Le hieros gamos d'Astarté». RSF 2, 1974 pp. 63-76; LIPISNKI, Edward, «Vestiges phéniciens d’Andalouise», OLP 15, 1984, pp. 81-132; VAN DER TOORN, Karel: «Female Prostitution in Payment of Vows in Ancient Israel», JBL 108, 1989, pp. 193-205; LIPINSKI, Dieux et Déesses..., op. cit., pp. 486-489, nota 418 con bibliografía; HENSHAW, op. cit., pp. 191-270, sobre todo 218 y ss.; JIMÉNEZ FLORES, Ana María. «Cultos fenicio-púnicos de Gadir: prostitución sagrada y puellae gaditanae», HABIS 32, 2001, pp. 11-29; RIBICHINI, Sergio, «Al servizio di Astarte. Ieroulia 
M.Yamauchi, la prostitución sagrada «is a practice involving the female and at times the male devotees of fertility deities, who presumably dedicated their earnings to their deity» $»^{40}$. Por su parte, Stephanie Budin la define como «the sale of a person's body for sexual purposes were some portion (if not all) of the money or goods received for this transaction belongs to a deity $»^{41}$.

Desde el punto de vista estrictamente literario, se haya documentada en todo el ámbito próximo oriental, aunque la descripción de la misma varía en función de unos textos u otros. En el Antiguo Testamento se hace una crítica importante de esta actividad, describiéndose incluso el atuendo de estas mujeres, las cuales se caracterizarían por su vestimenta así como por los adornos que sobre el pecho y la frente portaban ${ }^{42}$.

La inscripción de las cuentas de Kition comentadas anteriormente menciona a las servidoras del templo, cuya interpretación general ha sido la de prostitutas sagradas ${ }^{43}$. Incluso en la propia Cartago, algunas inscripciones en las que se mencionan a siervas de la divinidad o pertenecientes a una determinada congregación, han sido interpretadas como pertenecientes a congregaciones de prostitutas sagradas ${ }^{44}$. Generalmente, este tipo de actividades estaban asociadas a determinadas divinidades, como Astarté $e^{45}$, diosa de la fertilidad, entre otros atributos, donde la prostitución sagrada era realizada como parte del ritual de veneración de la deidad. Según los autores grecolatinos, las mujeres de los templos dedicados a esta diosa en Biblos y Sicca Veneria eran

e prostituzione sacra nei culti fenici e punici», en GonZÁlEz BLANCO, Antonio, MaTILLA SÉIQUER, Gonzalo y Alejandro EGEA VIVAnCOS (eds.), El mundo púnico. Religion, antropología y cultura material, Estudios Orientales 5-6, Murcia, 2004, pp. 55-68, sobre todo 62-64; JiMÉNEZ, La mano de Eva..., op. cit., pp. 91-97; BUDIN, Sacred Prostitution in..., op. cit., pp. 77-92; Budin, The Myth of Sacred Prostitution..., op. cit., pp. 10y ss.

40. YAMAUCHI, op. cit., p. 213. En su nota 3 aclara que la palabra griega hierodoulos, que significa esclava sagrada, es empleada en los textos clásicos para designar la prostitución sagrada femenina.

41. Budin, The Myth of Sacred Prostitution..., op. cit., p. 3.

42. Ribichini, Beliefs and Religious..., op. cit., p. 137.

43. MASSON y SZNYCER, op. cit., pp. 21-64; RIBICHINI, Beliefs and Religious..., op. cit., p. 136; RIBICHINI, Al servizio di Astarte..., op. cit., pp. 58-61; JIMÉNEz Cultos fenicio-púnicos..., op. cit., p. 19.

44. Ribichini, Al servizio di Astarte..., op. cit., pp. 55-56; LANCELlotTI, op. cit., p. 193.

45. Sobre esta diosa, muchos han sido los estudios que han analizado su presencia, atributos o su posible vinculación con la prostitución sagrada. Entre los trabajos dedicados a esta divinidad es menester destacar FANTAR, Mohammed Hassine: «A props d'Ashtart en Mediterranee occidentale». RSF 1, 1973, pp. 19-29; BONNET, Corinne, op. cit.; IZAK, Cornelius. The many faces of the goddess: the iconography of the Syro-Palestinian goddesses Anat, Astarte, Qedeshet, and Asherah c. 1500-1000 BCE, Fribourg- Göttingen, Academic Press- Vandenhoeck \& Ruprecht, 2004; RodRíGUEZ MuÑOZ, op. cit., pp. 26-28, con bibliografía. 
famosas ${ }^{46}$. En el santuario que dicha divinidad tenía en Erice también se mencionan a estas siervas de la divinidad, que tradicionalmente se han adscrito a la prostitución sagrada ${ }^{47}$.

Actualmente, como ya hemos comentado, el ejercicio de esta práctica está siendo cuestionada por la investigación, hasta tal punto de que algunos autores la niegan ${ }^{48}$, afirmando que sería una invención de la Biblia y los escritores clásicos que estarían encaminadas a desacreditar la religiosidad oriental. La arqueología no ha determinado la posibilidad de que este tipo de prácticas se llevaran a cabo desde un punto de vista estrictamente ritual. Las excavaciones realizadas en espacios cultuales no han permitido constatar datos que permitan afirmar el desarrollo de estos actos sagrados en ese recinto religioso. Lo único que ha podido documentarse es la posible adscripción de un espacio de culto a una determinada divinidad en función de la cultura material asociada al mismo y el sustento de las fuentes escritas y epigráficas. A pesar de ello, algunos autores han vinculado determinadas estructuras con esta práctica sagrada.

Un ejemplo podría ser la interpretación realizada por G. Colonna ${ }^{49}$ de las estancias localizadas en torno al santuario B de Pyrgi, de idénticas dimensiones $(2 \times 3 \mathrm{~m})$, quien las identificó como habitaciones en las que llevaba a cabo la prostitución sagrada, hipótesis que ha tenido gran aceptación en la investigación posterior gracias a un paisaje de Lucilio ${ }^{50}$. Esta práctica ha sido asocia-

46. LIPINSKI, Edward: Dieux et Déesses..., op. cit., pp. 487-488; LANCELlOTI, op. cit., pp. 193-194; RiBichini, Beliefs and Religious..., op. cit., pp. 131 y 137. Según éste último autor, en ellos la prostitución sagrada sería practicada por las propias esclavas del templo enmarcadas en una procesión ritual en honor de la diosa. También en RiBichini, Al servizio di Astarte..., op. cit., pp. 55-68.

47. CIS I, 3776. En AmadASI op. cit., p. 52. También Ribichini, Sergio, Beliefs and Religious..., op. cit., p. 133; RiBICHINI, Al servizio di Astarte..., op. cit., pp. 56-57.

48. Budin, Stephanie: The Myth of Sacred Prostitution..., op. cit., p. 1.

49. Colonna, Giovanni, «Il santuario di Pyrgi alla luce delle recenti scoperte». Studi Etruschi XXXIII, 1965, pp. 211-211; ColonNA, Giovanni. «Novità sui culti di Pyrgi». RendPontAcc 57, 1984-1985, pp. 59-64. Este autor afirmaba que «l'edificio in cui venita praticata la prostituzione sacra, da parte di sacerdotesse che, così facendo, si identificavano e si sostituivano alla dea, secondo l' antico rito orientale, che a Cipro ha dato origine al culto della Afrodite» (p. 64). Este santuario también ha sido estudiado por SERRA RIDGWAY, Francesca R.:»Etruscans, Greeks Carthaginians: The Sanctuary at Pyrgi», en Greek colonists and native populations: proceedings of the First Australian Congress of Classical Archaeology held in honour of emeritus professor A.D. Trendall, Canberra, New York, Oxford, Clarendon Press, Humanities Research Centre, Oxford University Press, 1990, pp. 511-530.

50. Para más información sobre los investigadores que aceptan esa hipótesis y los que se sitúan contrarios a ella, véase JiMÉNEZ, Cultos fenicio-púnicos..., op. cit., p. 22, nota 53; JiMÉNEZ, La mano de Eva..., op. cit., p. 94. 
da al culto a Astarté en función de unas láminas de oro en cuyas inscripciones se menciona a esta deidad ${ }^{51}$, lo que ha permitido vincular la participación de las mujeres en las prácticas cultuales dedicadas a esta divinidad femenina. Incluso se ha llegado a afirmar que este tipo de actividades se desarrollarían en santuarios costeros advocados a Melqart y Astarté, enclaves que no son únicamente centros religiosos sino también económicos, como parte de un ritual de hospitalidad a los extranjeros llegados al templo, actividades que se complementarían con danzas y otras diligencias artísticas ${ }^{52}$.

Desde el punto de vista iconográfico, también diversos autores han querido ver en los motivos de mujeres desnudas con peinados hathóricos documentadas sobre diversos soportes la representación de este tipo de «siervas». Tal es el caso de las representaciones documentadas en pendientes, colgantes, exvotos de bronce, etc ${ }^{53}$., todos ellos localizados en contextos cultuales, lo que, en un primer momento, podría avalar la relación de estas iconografías con el desarrollo de estas prácticas. Incluso la propia Astarté del Carambolo o las plaquitas de marfil que representan a una mujer en la ventana, parecen haber sucumbido a esta interpretación ${ }^{54}$.

Algunos autores señalaron en su momento la posibilidad de que la mujer, con independencia de su vinculación con el ámbito sacerdotal y religioso, también participara en el desarrollo de determinados ritos de paso que se hiciera extensible a todas las féminas que fueran a contraer matrimonio, lo que podría plantear la probabilidad de que ello fuera también una práctica común, aunque quizá sólo se desarrollara de modo temporal ${ }^{55}$.

También relacionado con el culto, sobre todo a divinidades femeninas, y pesar de las ya escasas referencias, durante el desarrollo de la práctica cultual podrían haberse realizado danzas rituales acompañadas de música por parte de las jóvenes que se encontraban al servicio de la divinidad. Según la Biblia, la danza ritual formaba parte de los cultos sirio-palestinos ${ }^{56}$. En Cádiz se

51. RIBICHINI, Beliefs and Religious..., op.cit., p. 134; BELFIORE, Valentina: «Studi sul lessico 'sacro': Laris Pulenas, le lamine di Pyrgi e la bilingue di Pesaro», Rasenna: Journal of the Center for Etruscan Studies 3, Iss. 1, Article 3, 2012, pp. 5-8. http://scholarworks. umass.edu/rasenna/vol3/iss1/3. Junto a Astarté, también se menciona a su equivalente etrusco, Uni.

52. RiBICHINI, Beliefs and Religious..., op. cit., p. 137; JIMÉNEZ, La mano de Eva..., op. cit., pp. 94-95.

53. Un resumen de todos estos objetos en JiMÉnEZ, La mano de Eva..., op. cit., pp. 93-94.

54. LIPISNKI, Edward: Vestiges phéniciens..., op. cit., p. 114; LANCELLOTTI, op. cit., p. 194.

55. YAMAUCHI, op. cit., p. 219; JimÉNEZ, Cultos fenicio-púnicos..., op. cit., p. 15; JiMÉNEZ, La mano de Eva..., op.cit., p. 92.

56. LIPISNKI, Dieux et Déesses..., op. cit., p. 485.

Feminismo/s 20, diciembre 2012, pp. 143-164 
llamaban puella $e^{57}$ a estas bailarinas ${ }^{58}$, las cuales, según esas fuentes, realizaban una danza de tipo sensual. Las cuentas del templo de Kition mencionan a cantantes entre el personal del mismo que posiblemente acompañarían dichas danzas sagradas ${ }^{59}$.

En este sentido, algunos autores han destacado la decoración de un cipo sardo de estructura piramidal que representa una escena de danza ritual en torno a un pilar de inspiración fálica en la que el papel protagonista de la actividad sagrada queda reservada al hombre, ataviado con faldilla y máscara de toro, mientras que las mujeres, desnudas, practican la danza de acompañamiento a dicho ritual ${ }^{60}$. Esta representación también la encontramos decorando algunas terracotas del templo B de Pyrgi comentado anteriormente, donde del mismo modo observamos a un personaje masculino ataviado con máscara en torno al cual se desarrolla una danza sagrada ejecutada por féminas ${ }^{61}$. Del mismo modo, la colección de cuencos de bronce y plata estudiados por G. Markoe en 1985 muestra varios ejemplos del desarrollo de actividades cultuales en los que la presencia femenina es destacada, como una parte importante de ese culto, decoración que también ha sido resaltada por la investigación ${ }^{62}$.

Peor conocido y, por ello, poco estudiado está la magia y las prácticas de adivinación que posiblemente se llevarían a cabo en algunos santuarios. De hecho, los documentos que pueden encuadrarse en la época de nuestro estudio se reducen a dos hechizos hallados en la región siria de Arslan Tash y su autenticidad no está exenta de polémica ${ }^{63}$. Posiblemente en los templos debió existir personal dedicado a la magia y la adivinación, prácticas muy importantes en las religiones próximo-orientales, pero no conocemos datos que nos informen de ello. Y menos aún de la presencia de la mujer en dichas prácticas.

57. Sobre las connotaciones de estas bailarinas en la historiografía española, véase OLMOS, Ricardo: « «Puellae Gaditanae»: ¿Heteras de Astarté?». AEspA 64, 1991, pp. 98-103.

58. Para R. Olmos, no se trataría de bailarinas sino de heteras, considerando a las puellae gaditanas como «el residuo o evolución profesionalizada de las originarias heteras de Astarté». En este sentido, véase, Olmos, op. cit., p. 108.

59. MASSON y SZNYCER, op. cit., pp. 21-68.

60. LIPISNKI, Dieux et Déesses..., op. cit., p. 486; JimÉNEZ, Cultos fenicio-púnicos..., op cit., p. 18 (para más bibliografía, ver nota 32 de esta autora).

61. VezÁR, Monika: «Pyrgi e l’Afrodite di Cipro», en Mélanges de l’Ecole française de Rome, Antiquitè 92, 1980, pp. 82-84; JiMÉNEZ, La mano de Eva..., op. cit., p. 94.

62. MARKoE, Glenn, Phoenician Bronze and Silver Bowls from Cyprus and the Mediterranean, Berkeley-Los Ángeles-London, 1985; JimÉNEZ, La mano de Eva..., op. cit., p. 95.

63. LIPINSKI, Dieux et Déesses..., op. cit., pp. 483-485, nota 399 con toda la bibliografía anterior; RiBICHINI, Beliefs and Religious..., op. cit., pp. 142-143; ZAMORA, José Angel: «Textos mágicos y trasfondo mitológico: Arslan Tash», en XeLlA, Paolo y José Ángel ZAMORA (eds.), Epigrafia e Storia delle religioni: Dal documento epigrafico al problema storico-religioso, SEL 20, Verona, 2003, pp. 9-23. 
Sí contamos con información sobre los oráculos de algunos santuarios, como el de Astarté en Cádiz ${ }^{64}$ dedicado a la misma diosa, como forma en la que se adivinaba el futuro y se solicitaba el favor de la divinidad, aunque esta información procede de las fuentes escritas.

Una vez expuesto el tipo de prácticas sagradas en los que la mujer participaría de un modo directo, nos restaría conocer a qué divinidades estaban vinculadas dichas prácticas con el fin de tratar de evidenciar la posible relación entre el papel de la mujer como colaboradora y participante de esos ritos y determinadas divinidades con atributos que recuerdan, precisamente, a los atributos femeninos por excelencia. Las fuentes con las que contamos para ello siguen siendo escasas. De hecho, únicamente existe una inscripción en la que se menciona explícitamente el nombre de una sacerdotisa y la divinidad a la que dedicaba culto. Se trataría de la inscripción de Ard-el-Kheraib comentada anteriormente, en la que se informa que Hanibaal era sacerdotisa de Koréé. El culto a Koré fue introducido oficialmente en Cartago, junto al de Deméter, en el 396 a. C. como consecuencia de la helenización de la ciudad, lo que repercutió en la instauración de un nuevo sacerdocio para este propósito ${ }^{66}$, sacerdocio en el que pudieron participar también las mujeres, tal y como indica esta inscripción. En relación con ello, y como ya señalara Sergio Ribichini:

these female deities are probably to be connected with fertility, prosperity, love and war; but we cannot establish with certainty, not even on the comparative level, whether there existed a relationship between the role attributed to then and the social position of woman at the time these cults developed, for we do not have sufficient data ${ }^{67}$.

Por todo lo anterior, parece ser que la participación de la mujer en la vida religiosa de la comunidad podría ir determinada en función del tipo de culto que allí se ejerciera. En este sentido, como hemos comentado a lo largo del presente artículo, y como también han resaltado otros investigadores, podríamos considerar que la administración del culto recaería en una fémina en aquellos santuarios que estuviesen advocados a una divinidad femenina

64. Para el caso gaditano contamos con la descripción que realiza Avieno, en su Ora Marítima, en la cual se indica textualmente «[...] hay una isla consagrada a Venus Marina, y en ella un templo con una cueva y un oráculo» (Or. Mar. 305-317).

65. BÉNICHOU-SAFAR, op. cit., pp. 216-217, no 49; JIMÉNEZ, El sacerdocio femenino..., op. cit., p. 16; JIMÉNEZ, La mano de Eva..., op. cit., p. 87.

66. Ribichini, Beliefs and Religious..., op. cit., p. 133. Sobre el culto de Deméter y Koré en Cartago, consultar PENA, María José: «El culto a Deméter y Core en Cartago. Aspectos iconográficos», Faventia 18/1, 1996, pp. 39-55; BONNET, Corinne: «Identité et altérité religieuses. À propos del’hellénisation de Carthage», Pallas 70, 2006, pp. 373-376.

67. RibICHINI, Beliefs and Religious..., op. cit., p. 122. 
cuyos atributos estuviesen vinculados con la mujer, como la fertilidad o la maternidad, con los cuales se sentirían plenamente identificadas. A ellas les estaría reservado el papel protagonista en el desarrollo de los mismos. No tenemos datos que nos permitan conocer si en aquellos santuarios advocados a una determinada divinidad donde su culto era administrado por una sacerdotisa o suma sacerdotisa, esa administración era compartida con sacerdotes masculinos. O si, por el contrario, las características y atributos de ese culto, claramente femeninos, impedían la entrada y participación del hombre en el mismo.

Desconocemos también si, en santuarios dedicados a otras divinidades, tanto los sacerdotes como las sacerdotisas, administraban culto de modo conjunto. Edward Lipinski determinaba que la actividad cultual de los templos fenicios era ejercida por sacerdotes y sacerdotisas, que eran auxiliados por lo que él define como un «clero» subordinado a ellos ${ }^{68}$. Con independencia o no de que compartamos esta moderna aceptación religiosa, creemos que tampoco contamos con información que nos indique si en todos los santuarios existía, además del sacerdote o sumo sacerdote, una sacerdotisa o jefa de las sacerdotisas que participara del algún modo en el desarrollo de los cultos que en el templo se llevaran a cabo. O si, por el contrario, la participación de la mujer como administradora de culto se reduce a aquellos santuarios en los que los atributos de las diosas están vinculados, como hemos comentado anteriormente, a aspectos relacionados con el ámbito femenino, estando exentas, por tanto, de jugar un papel importante en el resto de los santuarios advocados a otras divinidades. En este sentido, cobra especial importancia la cita de Silio Itálico en la que se informa sobre la exclusión de las mujeres en el templo gadirita de Melqart ${ }^{69}$.

En otro orden de cosas, Ana María Jiménez Flores indicaba que el modelo propuesto por Henshaw ${ }^{70}$ podría ser válido para el contexto en el que nos movemos, en función de la documentación epigráfica e iconográfica, incluyendo a las sacerdotisas dentro del primer grupo, a las bailarinas en el segundo, a las posibles magas y mujeres que realizaban profecías ${ }^{71}$ en el tercero, y a las prostitutas sagradas en el cuarto, grupo en el que también tendrían cabida las sa-

68. LIPINSKI, Dieux et Déesses..., op. cit., p. 456.

69. Silio Itálico, Pun. III, 21-22.

70. Véase notal7 del presente trabajo. HenSHAW, op. cit., p. 10.

71. Sobre la magia y la adivinación en el ámbito próximo oriental puede consultarse el libro de Ann Jeffers titulado Magic \& Divination in Ancient Palestine \& Syria, Leiden, E. J. Brill. De mismo modo, destacamos también su trabajo publicado en 2007 «Interpreting Magic and Divination in the Ancient Near East». Religion Compass, 1 (6), pp. 684-694. S. Ribichini le dedica un apartado especial en su estudio sobre las creencias 
cerdotisas y las bailarinas ${ }^{72}$. Algunos autores prefieren hacer otras distinciones en lo que a la posición de la mujer en el mundo religioso se refiere. Tal es el caso de Hennie J. Marsman, en cuyo estudio sobre la mujer en Ugarit e Israel diferencia entre las mujeres que simplemente adoran a una divinidad determinada y las especialistas religiosas, que se situaban entre la diosa y las adoradoras comentadas en primer lugar ${ }^{73}$, diferenciación que posiblemente también pueda aplicarse al ámbito de nuestro estudio, como una pervivencia del orden establecido en los siglos precedentes en la zona oriental, y que presumiblemente evolucionó hasta hacerse más complejo en el contexto occidental.

Con respecto a la polémica prostitución sagrada, las fuentes escritas que tradicionalmente se han consultado, y hoy se siguen haciendo, contienen un marcado sentido crítico que obliga a su interpretación y contextualización en los parámetros en los que dichas fuentes se redactaron. Los comentarios que aparecen en la Biblia contienen un excesivo contenido ético, marcado por un continuo juicio al politeísmo de la cultura fenicia en clara contraposición con la fe religiosa que se exalta. Y este contenido ético se extiende a la consideración que, por consiguiente, se manifiesta sobre la mujer fenicia, la cual es conceptualmente negativa desde la óptica de la tradición hebraica, que se muestra represiva con las costumbres de los pueblos orientales de su entorno más inmediato ${ }^{74}$.

Por su parte, la postura que encontramos en las fuentes clásicas suele caracterizarse por una crítica fundamentalmente ideológica, en la que subyace sobre todo la aversión política y cultural hacia lo extranjero. Por tanto, la crítica que se hace por parte de los escritores grecolatinos, a pesar de fabricar estereotipos que estigmatizarán a estos extranjeros, es, como señalan algunos investigadores ${ }^{75}$, menos feroz que la que encontramos en el Antiguo Testamento $^{76}$, sobre todo para el ámbito femenino, que es el que analizamos en el presente trabajo, aunque no por ello deja de ser una crítica.

A excepción de las fuentes escritas y epigráficas, cuyas interpretaciones, como vemos, no están exentas de polémica, no existen indicios arqueológicos

y la vida religiosa mencionado anteriormente. En este sentido, véase RIBICHINI, Beliefs and Religious..., op. cit., pp. 142-144.

72. JiMÉNEZ, La mano de Eva..., op. cit., pp. 98-99.

73. Marsman, Hennie J.: Women in Ugarit and Israel. Their social and Religious Position in the Context of the Ancient Near East, Leiden, E.J. Brill, 2003, p. 476.

74. LANCELlOTTI, op cit., p. 189.

75. Ibidem.

76. Sobre la información aportada por la Biblia y la crítica a las poblaciones orientales circundantes, véase XELlA, Paolo: «La Bible», en KRINGS, Véronique (ed.), La Civilisation Phenicienne et Punique, E. J. Brill, The Netherlands, 1995, pp. 64-72. 
ni iconográficos claros que nos permitan vincular esta práctica al culto de determinadas divinidades, como parte del desarrollo de ese culto. El propio Edwin M. Yamauchi resaltaba en su trabajo que, a pesar del hallazgo de numerosas representaciones de mujeres desnudas en las placas halladas en Ugarit, no hay referencia de esta práctica en los textos ugaríticos ${ }^{77}$, basando su conocimiento únicamente en la documentación aparecida en los textos greco-latinos. Y lo mismo sucedía para el caso de Cartago, por ejemplo, cuya evidencia también es ambigua.

Para el caso de Pyrgi, la identificación que se hace de las habitaciones sagradas se basa únicamente en testimonios escritos que asociaban el lugar a la prostitución sagrada en honor a Astartét ${ }^{78}$. De hecho, algunos investigadores, como Cristofani, han planteado otras interpretaciones para estas estancias, a las que considera almacenes o tiendas ${ }^{79}$.

Por otro lado, tradicionalmente, algunas inscripciones, que mencionan a hijas núbiles o siervas de Astarté, han sido identificadas como prueba del desarrollo de esta práctica ${ }^{80}$. Creemos que no es del todo acertada la interpretación que se hace de siervas entendidas como prostitutas sagradas. La categoría de sierva dentro de un culto no tiene por qué llevar aparejado el desarrollo de la prostitución. En este sentido, lo que nos interesa destacar es que no cuestionamos que existiese la prostitución en el ámbito de los santuarios, sino que creemos que no era una actividad única y exclusiva de este ámbito. Consideramos que la prostitución era una práctica que posiblemente se podría haber llevado a cabo en otros contextos como el cortesano o político, además del religioso, por lo que no estamos seguros de poder compartir la tradicional teoría de asociar esta actividad al culto de determinadas divinidades, entendiéndola como sagrada y como parte de ese ritual. Más factible, por tanto, nos parecería interpretar a estas mujeres como devotas que llevarían a cabo tareas relacionadas con la adoración de la divinidad y el mantenimiento del templo, aunque, lamentablemente, no tenemos datos que nos indiquen cuales serían las funciones de las mismas ${ }^{81}$.

Parece claro, en tal caso, que es necesario llevar a cabo un replanteamiento de las tradicionales teorías sobre la cuestión de la mujer dentro del ámbito cultual fenicio-púnico, superando las viejas connotaciones que la historiografía

77. YAMAUCHI, op. cit., p. 219.

78. Budin, Stephanie: The Myth of Sacred Prostitution..., op. cit., pp. 247-254.

79. CRISTOFAnI, Mauro: «Ripensando Pyrgi». Miscellanea Ceretana I (Quaderni del Centro di Studio per lá archeologia etrusco-italica 17), 1989, pp. 92.

80. LIPINSKI, Dieux et Déesses..., op. cit., pp. 487.

81. VIDAL, Jordi: Op. cit., p. 89. 
ha vinculado a las féminas amparadas por las siempre cuestionadas fuentes y justificadas bajo el sesgo de lo sagrado. Actualmente parece plausible la existencia de un papel protagonista para un grupo exclusivo de mujeres, así como un papel destacado para el resto en lo que a la religiosidad se refiere, con una diversificación funcional que abarcarían aspectos tan importantes como la danza, la música, la adivinación, las tareas de mantenimiento del templo o la ayuda prestada a las sacerdotisas en la administración del propio culto. Creemos que este puede resultar un ámbito de trabajo muy importante que inevitablemente servirá para poner en duda muchas de las afirmaciones comúnmente aceptadas por la investigación.

\section{Abreviaturas}

CIS: Corpus Inscriptionum Semiticarum. Paris 1881-1886.

KAI: H. Donner-W. Röllig, Kanaanäische und aramäische Inschriften. Wiesbaden 1966-1969.

\section{Referencias bibliográficas}

AMADASI GUZzo, Maria Giulia:» Il sacerdote», en ZAMORA, José Ángel (ed.): El hombre fenicio: estudios y materiales, Roma, CSIC, 2003, pp. 45-53.

BeLFIORE, Valentina: «Studi sul lessico 'sacro': Laris Pulenas, le lamine di Pyrgi e la bilingue di Pesaro». Rasenna: Journal of the Center for Etruscan Studies 3, Iss. 1, Article 3 (2012), pp. 5-8. http://scholarworks.umass.edu/rasenna/vol3/ iss $1 / 3$

BÉNICHOU-SAFAR, Hélène. Les Tombes Puniques de Carthage. Topographie, structures, inscriptions et rites funéraires, Paris, CNRS, 1982.

Bird, Phyllis: 'The Place of Women in the Israelite Cultus', en Miller, Patrick D., HANSON, P. D., McBride S. Dean (eds.): Ancient Israelite Religion: Essays in Honor of Frank Moore Cross, Philadelphia, Fortress Press, 1987, pp. 397-420.

BISI, Anna Maria. «Le stele puniche di Lilibeo e il problema dell'influenza semitica nella religione e nell'arte della Sicilia Occidentale». Karthago XIV (1968), pp. 227-234, tav. I-V.

Bonnet, Corinne. Astarté. Dossier documentaire et perspectives historiques, Roma, Consiglio Nazionale delle Ricerche. 1996.

BONNET, Corinne: «Identité et altérité religieuses. À propos del'hellénisation de Carthage». Pallas 70 (2006), pp. 365-379.

Budin, Stephanie Lynn: «Sacred Prostitution in the First Person», en FARAONE, Christopher, MCCluRE, Laura (eds.): Prostitutes and Courtesans in the Ancient World. Madison. 2006, pp. 77-92; 
-, The Myth of Sacred Prostitution in Antiquity, New York, Cambridge University Press. 2008.

CAubet, Annie: «Les sanctuaires de Kition à l'époque de la dynastie Phénicienne», Religio Phoenicia, Studia Phoenicia IV, Namur, 1986, pp. 153-168.

Colonna, Giovanni. «Il santuario di Pyrgi alla luce delle recenti scoperte». Studi Etruschi XXXIII (1965), pp. 211-212.

—, «Novità sui culti di Pyrgi». RendPontAcc 57 (1984-1985), pp. 59-64.

Cristofani, M.: «Ripensando Pyrgi». Miscellanea Ceretana I (Quaderni del Centro di Studio per lá archeologia etrusco-italica 17), 1989, pp. 85-93.

DE Geus, Cornelius H. J.: «The material culture of Phoenicia and Israel», en

LIPINSKI, Edward (ed.): Phoenicia and the Bible, Studia Phoenicia XI, Leuven, Orientalia Lovaniensia Analecta 44, 1991, pp. 11-16.

DELCOR, Mathias. «Le hieros gamos d’Astarté». RSF 2 (1974), pp. 63-76.

ELAYI, Josette: «Le roi et la religion dans les cités phéniciennes à l'époque perse», en Religio Phoenicia, Studia Phoenicia IV, Namur, 1986, pp. 249-261.

FANTAR, Mohammed Hassine: «A props d'Ashtart en Mediterranee occidentale». RSF 1 (1973), pp. 19-29.

Henshaw, Richard A. Female and Male. The cultic Personnel: the Bible and the Rest of the Near East, Allison Park, P A: Pickwick Publications, 1994.

IZAK, Cornelius. The many faces of the goddess: the iconography of the Syro-Palestinian goddesses Anat, Astarte, Qedeshet, and Asherah c. 1500-1000 BCE, Fribourg- Göttingen, Academic Press- Vandenhoeck \& Ruprecht, 2004.

JefFers, Ann. Magic E Divination in Ancient Palestine $\&$ Syria, Leiden, E. J. Brill.

-, «Interpreting Magic and Divination in the Ancient Near East». Religion Compass 1 (6) (2007), pp. 684-694.

JimÉnez Flores, Ana María. «Cultos fenicio-púnicos de Gadir: prostitución sagrada y puellae gaditanae». HABIS 32 (2001), pp. 11-29.

—, «El sacerdocio femenino en el mundo fenicio-púnico». SPAL 11 (2002), pp. 9-20.

—, «La mano de Eva: las mujeres en el culto fenicio-púnico», en EsCACENA CARRASCO, José Luis, FERRER AlBELDA, Eduardo (eds.): Entre dioses y los hombres: el sacerdocio en la antigüedad. Sevilla, SPAL Monografías VII, 2006, pp. 83-102.

-, y MARÍN CEBAllos, María Cruz: «Jerarquía social y sacerdocio fenicio-púnico: apuntes sobre su relación», en Hernández Guerra, L., Alvar EzQUerra, Jaime (eds.): Jerarquías religiosas y control social en el mundo antiguo. Actas del XXVII Congreso Internacional Girea-Arys IX, Valladolid, 2004, pp. 80-86.

LANCELlOTTI, Maria Grazia: «La donna», en ZAMORA, J. A. (ed.): El hombre fenicio. Estudios y materiales, Roma, CSIC, 2003, pp. 187-197.

LIPINSKI, Edward. «Vestiges phéniciens d'Andalouise». OLP 15 (1984), pp. 81-132. 
—, Dieux et Déesses de l’únivers phénicienne et punique. Leide, 1995.

MACURDY, Grace H. Hellenistic Queens. A study of women-power in Macedonia, Seleucid Syria and Ptolemaic Egyp, The John Hopkins Press, Baltimore, 1932.

MAES, Annemie: «Le costume phénicien des stèles D`Umm el-Amed», en LIPINSKI, Edward (ed.): Phoenicia and the Bible, Studia Phoenicia XI, Orientalia Lovaniensia Analecta 44, Leuven, 1991, pp. 209-230.

MARKoE, Glenn. Phoenician Bronze and Silver Bowls from Cyprus and the Mediterranean. Berkeley-Los Ángeles-London, 1985.

MARSMAn, Hennie J.: Women in Ugarit and Israel. Their social and Religious Position in the Context of the Ancient Near East, Leiden, E.J.Brill, 2003.

MASSON, Olivier, SZNYCER, Maurice: Rechercher sur les Phéniciens à Chypre, Ginebra- Paris, Centre de Recherches d'Histoire et de Philologie, 1972, pp. 21-68.

MERLIN, Alfred; DrAPPIER, Louis. La necropole punique d'Ard El-Kheraiö á Carthague (Notes et documents publiès par la Direction des Antiquités de Tunis. III). Paris, 1909.

Mirón PÉREZ, María Dolores: «Mujeres y poder en la Antigüedad Clásica: Historia y Teoría Feminista», Saldvie 10 (2010), 113-125.

Olmos, Ricardo: ««Puellae Gaditanae»: ¿Heteras de Astarté?». AEspA 64 (1991), pp. 99-109.

PenA, María José: «El culto a Deméter y Core en Cartago. Aspectos iconográficos». Faventia 18/1 (1996), pp. 39-55.

PUECH, Emile. «Un cratère phénicien inscrit: rites et croyances». Transeuphatène 8 (1994), pp. 47-73.

RibiCHINI, Sergio: Al servizio di Astarte. Ieroulia e prostituzione sacra nei culti fenici e punici», en González Blanco, Antonio, Matilla SÉIQuer, Gonzalo, EGEA Vivancos, Alejandro (eds.): El mundo púnico. Religion, antropología y cultura material, Estudios Orientales 5-6, Murcia, 2004, pp. 55-68.

-, «Beliefs and Religious Life», en Moscati, Sabatino (ed.) The Phoenicians. London-New York, I. B. Tauris, 2001, pp. 120-153.

RodríGUEZ MuÑoz, Raquel: «El uso cúltico del agua en el mundo fenicio y púnico. El caso de Astarté en Cádiz». Herakleion 1 (2008), pp. 21-40.

RUIZ CABRERO, Luis Alberto: «Sociedad, jerarquías y clases sociales de Cartago», en COSTA, Benjamin; FERNÁNDEZ, Jordi H. (eds.): Instituciones, demos y ejército en Cartago, TMAEF 64, 2009, pp. 7-73.

SERRA RidGWAY, Francesca R.:»Etruscans, Greeks Carthaginians: The Sanctuary at Pyrgi», en Greek colonists and native populations: proceedings of the First Australian Congress of Classical Archaeology held in honour of emeritus professor A.D. Trendall, Canberra, New York, Oxford, Clarendon Press, Humanities Research Centre, Oxford University Press, 1990, pp. 511-530.

VAN DER TOORN, Karel: «Female Prostitution in Payment of Vows in Ancient Israel». JBL 108 (1989), pp. 193-205. 
VEzÁR, Monika: «Pyrgi e l'Afrodite di Cipro», en Mélanges de l'Ecole française de Rome, Antiquitè 92, 1980, pp. 35-84.

VIDAL, Jordi. «Ummahnu, sierva de la Señora de Biblos. Apuntes prosopográficos». Aula Orientalis 28 (2010), pp. 85-92.

Williams, Mary Gilmore. «Studies in the lives of Roman Empresses, I: Julia Domna». American Journal of Archaeology 6 (1902), pp. 259-305.

Xella, Paolo: «La Bible», en Krings, Véronique (ed.): La Civilisation Phenicienne et Punique, E. J.Brill, The Netherlands, 1995, pp. 64-72.

YAmauchI, Edwin Maseo: «Cultic Prostitution», Orient and Occident. Essays presented to Cyrus H. Gordon on the Occasion of his Sixty-fith Birthday, AOAT 22, Kevelaer-Neukirchen-Vluyn, 1973, pp. 213-222.

Yon, Marguerite: «Le «Maître de l'eau» à Kition», en Archéologie au Levant. Recueil R. Saidah, Collection de la Maison de l'Orient Méditerranéen 12, Série archéologique 9, Lyon, 1982, pp. 251-263.

ZAMORA, José Ángel: «Textos mágicos y trasfondo mitológico: Arslan Tash», en Xella, Paolo; Zamora, José Ángel (eds.): Epigrafia e Storia delle religioni: Dal documento epigrafico al problema storico-religioso (=SEL 20), Verona, 2003, pp. 9-23.

—, «El sacerdocio en el Levante próximo-oriental (Siria, Fenicia y el mundo púnico): las relaciones entre el culto y el poder y la continuidad en el cambio», en Escacena Carrasco, José Luis; Ferrer Albelda, Eduardo (eds.): Entre dioses y los hombres: el sacerdocio en la antigüedad, SPAL Monografías VII, Sevilla, 2006, pp. 57-82. 